ISSN: 2582-0435

Case Report | Vol 4 Iss S9

\title{
Effectiveness and Safety of Physical Therapy Intervention in Adult Dermatomyositis: A Case Report and Review
}

\author{
Isha Akulwar-Tajane \\ Department of Physiotherapy, KJ Somaiya College of Physiotherapy, Mumbai, India \\ Correspondence should be made to Isha-Akulwar Tajane, $\underline{\text { drishasa@yahoo.co.in }}$
}

Received: 15 January 2021; Accepted: 20 February 2021; Published: 27 February 2021

\begin{abstract}
\section{BACKGROUND}

The evidence for positive effects of exercise is insufficient in dermatomyositis. This article aims to outline the effects of physical exercise with regard to structure (Disease activity, inflammation, metabolic milieu and muscle structure) as well as clinical outcome in terms of impairment, activity limitation, participation restriction according to the ICF; and QOL in an adult patient with dermatomyositis.
\end{abstract}

\section{CASE DESCRIPTION}

A 47-years old female diagnosed with definite DM since last 10 months was referred by a Rheumatologist for physical therapy intervention.

\section{INTERVENTION}

12 weeks (Three days a week) of Physiotherapy (Moderate intensity, individualized, closely monitored) along with a structured home exercise program (Low intensity, 5 days - 6 days a week).

\section{OUTCOME MEASURES}

Assessment of muscular pain, muscle strength, endurance, balance, functional evaluation, and QOL along with serological investigations.

\section{RESULTS}

\section{Effectiveness}

Patient continued to show meaningful and clinically significant improvement on standardized outcome measures:

1. A major clinical response (total improvement score of 77) on IMAC S core set measures.

2. Improvement across all domains of ICF viz. muscle strength; endurance; movement coordination; balance; aerobic endurance; confidence and performance in activities of daily living; participation in social activities.

Citation: Isha Akulwar-Tajane, Effectiveness and Safety of Physical therapy Intervention in Adult Dermatomyositis: A Case Report and Review. J Clin Cases Rep 4(S9): 5-20. 
http://www.tridhascholars.org | June-2021

3. Probable disease-modifying effect: Progressively tapering dose of prescribed corticosteroids medication and improvement in disease-specific biochemical markers.

4. Patient reported measures: Reduction in muscular pain, shortness of breath \& fatigue; and improvement in Healthrelated QOL.

\section{SAFETY}

(As evident clinically and from the investigations):

1. No adverse reaction to exercises or physical activity.

2. Remained in remission - stable disease activity.

\section{CONCLUSION}

Moderate intensity of physiotherapy intervention along with home exercise program is effective and safe in improving functional outcomes for dermatomyositis in a stable disease activity state. This case report enhances our understanding of the effects of physical exercise and may improve treatment and outcome in patients with dermatomyositis.

\section{KEYWORDS}

Dermatomyositis; Physical exercise; QOL

\section{ABBREVIATIONS}

IIM =Idiopathic Inflammatory Myopathies; DM = Dermatomyositis; PM = Polymyositis; WHO = World Health Organisation; $\mathrm{ICF}=$ International Classification of Functioning, Disability and Health; QOL = Quality Of Life; MMT = Manual Muscle Testing; VAS = Visual Analogue Scale; ADL = Activities of Daily Living; IMAC S = International Myositis Assessment and Clinical Studies Group; MyoAct = Myositis Disease Activity Assessment VAS; MITAX = Myositis Intention-to-Treat Activity Index; EULAR/ACR = European League Against Rheumatism/American College of Rheumatology

\section{INTRODUCTION}

The idiopathic inflammatory myopathies (IIM), collectively known as myositis are a heterogeneous group of systemic autoimmune diseases that primarily affect skeletal muscles [1]. Traditionally, IIMs are classifiable in three subtypes, polymyositis (PM), dermatomyositis (DM), and inclusion body myositis (IBM), but recently, also other subgroups, such as necrotizing autoimmune myopathy (NAM) and anti-synthetase syndrome have been identified [2]. Dermatomyositis (DM) involves a characteristic skin rash and is characterized by reduced muscle strength, fatigue and myalgia. This is a rare condition with the estimated incidence of 1 case - 9 case per million people [3]. Diagnosis is according to the EULAR/ACR criteria which combine clinical, laboratory, and pathologic features [4]. It is coded in ICD - 10 as M33.10 [5].

IIMs are considered as potentially treatable myopathies [6]. Standard medical treatment consists of oral corticosteroids in combination with immunosuppressive treatment. Although responding to treatment, complete recovery of muscle function with pharmacological treatment does not always occur. Muscle strength, functional status and quality of life remain impaired and a majority of patients develop sustained disability, suggesting that physical inability is a great concern for these patients $[7,8]$. In this context, Physical therapy is usually indicated to prevent muscle atrophy; and to regain muscle strength and range of motion. Over the last two decades studies evaluating active exercise in IIM support the notion of safety and benefits of exercise and indicate 
that active exercise, adapted to disease activity and disability should be included in the rehabilitation of patients in all stages of IIM. However, a cochrane review [9] in 2013 of five randomized controlled trials (RCTs) of exercise in muscle disease, including one study with patients with polymyositis (PM) or (DM), demonstrated that the evidence for positive effects of exercise is insufficient due to rarity of this disorder, its complex clinical phenotypes, and small number of trials included, their risk of bias and lack of control group. Although effectiveness of exercise on muscle functions have been reported, its efficacy on disability and quality of life has not been proved and also its efficacy in terms of newly developed and validated outcome measures has not yet been established. Many clinical trials are currently registered and recruiting participants, however these have combined DM and PM. DM and PM are clinically, histologically and pathogenically distinct entities. Associated symptoms and physical findings may vary widely from case to case as patients may present differently. There are no standardized therapeutic guidelines for treatment of IIM and the therapeutic approach is mainly guided by expert opinion and case series. More studies are required to establish the safety and benefits of different types of exercise. The aim of this case report is to outline the effects of physical exercise with regard to structure (disease activity, inflammation, metabolic milieu and muscle structure) as well as clinical outcome in terms ofimpairment, activity limitation, participation restriction according to the ICF, and QOL in an adult patient with DM.

\section{CASE REPORT}

A 47-years old female diagnosed with DM since last 10 months (June 2018) was referred by a rheumatologist for physical therapy intervention. She reported with muscle weakness in the trunk, around pelvic girdle \& hip region (difficulty in walking, climbing stairs, getting up from squatting position and a seated position from ground); myalgia (especially in the neck and trunk region); dyspnea, fatigue; and marked skin changes over trunk and face.

Medical history, investigations and treatment history was noted. Patient was having multiple systemic and other unspecific symptoms since last 7 years however she was never suspected and thus was never investigated for DM and was undiagnosed for several years. In June 2018, patient developed acute skin changes characteristic of DM, muscular manifestations. Other symptoms were dysphagia, shortness of breath and dry cough. Investigations for initial diagnosis essentially showed signs of muscle inflammation with elevated levels of muscle enzymes andmyositis-specific autoantibodies on serologic evaluation. EMG investigation was normal and muscle biopsy was not done. A diagnosis of definite DM with myopathic subtype was then established for her. Also she was clinically diagnosed with an overlap syndrome SJogren syndrome (though specific blood test done during acute flare of symptoms was negative). Patient was immediately started on high dose corticosteroid medication (oral and in divided doses) and corticosteroidsparing agent. She underwent serological investigations periodically for monitoring her disease activity and response to immunosuppressive and immune-modulating agents.

\section{Physical Examination}

Her higher mental functions, cranial nerve testing and sensory examination was unremarkable), patient was evaluated using The International Myositis Assessment Clinical Study Group (IMACS) Core set of disease measures [10] as mentioned in [Table 1]. The use of this validated measure has been recommended to assist in standardizing outcome measurement and in optimizing therapeutic trials in myositis. It includes assessments of disease activity; muscle strength; physical function; serum activity of muscle enzymes; and an assessment tool to capture extra-skeletal muscle disease activity. Disease 
activity in myositis refers to the type, extent and severity of reversible manifestations due to myositis [11]. Disease damage is defined as persistent changes in anatomy, physiology, pathology or function, which are present for at least 6 months $[10,11]$. Damage may be the result of prior active disease (causing scarring, fibrosis and atrophy), complications of therapy, co-morbid conditions, or other events. A portion of disease damage is disease chronicity. Definition of improvement is given as "any 3 of 6 core set measures improved $\geq 20 \%$ with no more than 2 (not including MMT) worse by $\geq 25 \%$ " [10]. Also, the degree of change in each core set measure that is clinically meaningful is defined. For the domains of muscle strength and physical function, a minimum of $15 \%$ improvement is clinically significant, whereas for the physician and patient global assessments, as well as the extra-muscular assessment, a minimum of $20 \%$ improvement is considered clinically meaningful, and for serum levels of muscle associated enzymes, at least $30 \%$ improvement is needed to be clinically important. $\geq 20 \%$ improvement is consensus of clinically meaningful change. The total improvement score is the sum of all 6 improvement scores associated with the change in each core set measure.

Muscle strength is central to the assessment of myositis patients. It was assessed clinically using MMT. Patient presented with marked muscle atrophy and weakness in the axial and proximal muscles symmetrically, notably in the hip flexors, extensors, abductors, neck flexors and shoulder abductors. She had considerable difficulty in recruiting abdominals with poor sustenance (less time and weak hold). Movement control and whole body coordination was impaired in presence of significant muscle weakness. She demonstrated weak hip and trunk postural control strategies. Her Berg balance scale score was 53 (moderate risk of falls). She had no history of fall, however reported 'fear of fall'. She had self-imposed limitations on physical activities due to lack of confidence during movement transitions. 'Shivering', 'wobbling' was the subjective feeling reported by her during walking.

She reported pain in thoraco-lumbar region which was not related to any particular movement or activity and occasional soreness in leg muscles. This symptom often used to wax and wane for no apparent reason.

Dyspnea as reported on a 10 point scale was 8 (on activity, sometimes even at rest).

\section{Functional Evaluation}

Patient was independent in bed mobility, transfers and in sit to stand but with difficulty. She could walk on overhead ground, however preferred assistance for walking indoor and outdoor. Her functional mobility as assessed on Timed up and Go test was 14.2 seconds. For the staircase she requited mechanical support of railing as well as manual assistance. More than actual weakness, her fear of fall was predominantly contributed with unpredictable shivering, wobbling of the legs. Despite of having some weakness in her upper limb muscles, she was independent for all upper limb activities. Patient experienced significant fatigue and shortness of breathwhich interfered with her ADL.

\section{Disability}

On modified rankin scale [12] (a global assessment of disability) patient graded her disability as 4 (described as 'unable to attend to own bodily needs without assistance, and unable to walk unassisted') which is considered as moderately severe disability.

Health-related QOL was measured with the selfadministered questionnaire SF-36 [13], which contains questions relating to self-experienced health in eight dimensions: Physical functioning, physical role, bodily pain, general health, vitality, social functioning, emotional role and mental health. Patient reported poor perceived health across all dimensions. 


\section{Psychological Status}

Patient reported episodes of anxiety, feelings of selfhelplessness and disability. Her concerns were associated with prolonged (almost for 7 years) physical and psychological suffering before the diagnosis and treatment; uncertainty of the disease course; life-long need for medication; dependency in ADL and significant functional disability; impaired quality of life; dealing with menopause, etc.

Her medical condition seemed to be stable. Supervised exercise program (to closely monitor response to exercises and prevent muscular injuries and to exercise at an optimal level) on regular basis was recommended.

\section{Intervention}

Patient received 12 weeks (three days a week on nonconsecutive days) of Physiotherapy (individualized, closely monitored) along with a structured home exercise program (low intensity, 5 days - 6 days a week).

Exercise program started on a lower intensity, gradually increasing to goal intensity during the first two weeks. Gradually the intensity and duration of exercise was increased to a rate of exertion that the patient found somewhat challenging but not overly difficult. Physiotherapist reviewed her expectations, prognosis, and progress on a regular basis and attempted to set realistic goals based on continued discussion.

The physical therapy plan of care focused on strengthening and stretching, with an emphasis on a home exercise program. Types of exercises included: strengthening; flexibility exercises; movement control and coordination training; postural control and balance training; aerobic endurance training; thoracic mobility and breathing exercises; task-specific functional training; ergonomic advice and activity pacing. Her physiotherapy protocol included: Each exercise session started with 10 minutes warm-up, stretching. Exercises to improve muscle strength and function targeted the trunk, pelvic girdle, hip and knee muscles and included active and resistance exercises adapted as per the degree of muscle weakness. These included progressive resistance training (moderate intensity) with therabands and also functional exercises (sit-to-stand training, reaching for weights on multi-level shelving). A detailed strength examination at every visit wasdone for assessing treatment response and strengthening program was adapted accordingly. Flexibility exercises included muscle lengthening, range of motion, and stretching with relaxation and breathing incorporated. In movementcontrol and coordination training, large segment stability and mobility muscles were targeted. Postural control and balance training was given in various functional upright positions to improve postural awareness and postural strategies (both anticipatory and reactive). Task -specific functional exercises included sit to stand training and gait training on level surfaces and also stair - case training was started later. In view of negative effects of corticosteroid medication on bone health, low impact weight bearing exercises were incorporated. Thoracic mobility and Breathing exercises were practiced intermittently in upright positions with various upper limb movements. Aerobic endurance conditioning exercises started with Initial three weeks of lower extremity stationary cycling on a bike (on day one - terminated only after 1.11 seconds due to shortness of breath, later progressed to 10 minutes of continued cycling). Treadmill training was started after three weeks and at a minimum speed of $0.8 \mathrm{~km} /$ hour for 5 minutes, progressively 2 minutes - 5 minutes were added subsequently for every session and progressed to 20 minutes of continued walking at a speed of $4 \mathrm{~km} / \mathrm{hour}$. Each therapy session ended with stretching of trained muscle groups and rating of overall exertion during the exercise session on the Borg RPE-scale. Owing to her limited endurance for exercises- multiple goals were incorporated into the same exercise regime viz. strengthening in functional patterns involving multiple 
segments and in upright positions thus simultaneously improving movement coordination and balance. A home exercise program was designed by incorporating similar principles as that of the supervised therapy program. The patient performed exercises at home on an easy to moderate intensity adapted to initial muscle weakness, five days a week for 12 weeks. The program consisted of stretching exercises; breathing exercises; strengthening exercises for the trunk and pelvic girdle muscles; seated dynamic knee-extensor exercise; biceps and deltoid curls; seated rowing; heel and toe lifts; whole-body rising from sitting to standing; kneel walking, etc. The program was divided into two/more sessions with adequate rest in between and took about 30 minutes to perform. She filled out an exercise diary commenting on loads and number of repetitions for each task. Progressively during the course of therapy patient was advised for outdoor walks of 20 minutes.

\section{Monitoring}

Findings, tolerance and response to physiotherapy was monitored on daily basis and changes were made accordingly. Before exercise session patient used to rate self-reported pain and fatigue on a visual analogue scale and describe in her own words information about possible changes in medication dose, any side effects from medication as well as possible negative and positive effects from previous exercise sessions, as well as physical activities performed since the previous exercise session. She filled out an exercise diary for HEP and her physical activity log. Borg 6 RPE - 20 RPE scale was used every day to monitor the intensity of exercise program. Thus, exercise was individually adapted to disease activity, medication dose and especially to the level of pain and fatigue. Patient showed good compliance to supervised therapy program and HEP.

Ergonomic advice was given for ADLs and advice on activity pacing to reduce fatigue and improve endurance. She was advised for some environmental modifications at home for reducing functional limitations such as use of grab bars for the bath, shower, and raised toilet seats.Patient was instructed that during acute flare up, exercises to be discontinued or reduced to gentle isometrics and ROM exercises. Avoid additional exercises and physical activity was restricted to daily functional mobility and walking. Also, for HEP she was told not to exercise on any given day if experiencing fatigue or pain from the previous day. Advice was given on dietary consultation.

\section{RESULTS}

\section{Effectiveness}

Patient continued to show meaningful and clinically significant improvement on standardized outcome measures over a period of 12 weeks.

Patient showed improvement above the threshold for minimal important clinical change for the entire core set measures. Her total improvement score was 77 which corresponds to major clinical response $(>60=$ Threshold for major clinical response as per ACR/EULAR criteria), 2016 [14].

\begin{tabular}{|c|c|c|c|c|}
\hline Core Set Domain & Scale & Baseline & At 12 Weeks & Change \\
\hline Physician Global Disease Activity & Likert scale (0-5) & 2 (moderate) & 1 (Mild) & $20 \%$ \\
\hline Patient Global Activity & VAS (0-10) & 4.3 & 1.2 & $31 \%$ \\
\hline \multirow{4}{*}{ Muscle Strength } & MMT $24(0-10)\{$ Out of 260$\}$ & 146 & 199 & $20.4 \%$ \\
\hline & Axial (2) $\{$ Out of 20$\}$ & 10 & 14 & \\
\hline & Proximal $(8 \times 2)\{$ Out of 60$\}$ & 88 & 125 & \\
\hline & Distal $(4 \times 2)\{$ Out of 80$\}$ & 24 & 36 & \\
\hline Physical Function & HAQ (0-3) & 2 & 1.3 & $23.33 \%$ \\
\hline Muscle Enzymes & \multicolumn{3}{|c|}{$\geq 2$ enzymes $(\mathrm{CPK}$, SGOT, LDH) } & $29.14 \%$ \\
\hline \multirow{2}{*}{ Extra Muscular Activity } & Myo Act $\{$ Out of 60$\}$ & 22.4 & 6.5 & $26.4 \%$ \\
\hline & MITAX $\{$ Out of 63$\}$ & 32 & 24 & $12.70 \%$ \\
\hline Total Improvement Score & \multicolumn{4}{|c|}{$77(>60=$ Threshold for major clinical response as per ACR/EULAR criteria, 2016) } \\
\hline
\end{tabular}


Table 1: IMACS core set measures.

At the beginning patient required high dose corticosteroid with other immunomodulatory therapy. After intervention was started, along with physician and patient reported improvement in global disease activity (as mentioned in the table above), her physician prescribed progressively tapering dose of corticosteroids medication which was not associated with relapse of symptoms.

\section{Patient-Reported Outcomes}

1. Pain in thoraco-lumbar and pelvic region subsided completely.
2. Improvement across all components of Health-related Quality Of Life (SF -36).

3. Sleep patterns and quality of sleep improved.

4. Commented that "I feel now I am living in my body".

\section{Safety - (As Evident Clinically and from her Investigation Reports)}

Overall the physical therapy and home exercise training program was well tolerated by the patient. Patient showed better control of disease activity, positive response to therapy with no adverse reaction, tolerance to exercise and remained in stable disease condition (Table 2).

\begin{tabular}{|c|c|c|}
\hline ICF Domain & Parameter & Outcome \\
\hline \multirow{5}{*}{$\begin{array}{l}\text { Body Structure } \\
\text { and Function }\end{array}$} & $\begin{array}{l}\text { Muscle } \\
\text { Strength }\end{array}$ & Improved grade on MMT \\
\hline & $\begin{array}{c}\text { Muscle } \\
\text { Endurance }\end{array}$ & Increased sustenance- (time and strength of hold) \\
\hline & $\begin{array}{c}\text { Motor } \\
\text { Coordination } \\
\end{array}$ & Better initiation and sustenance of movements and better multi-segment control \\
\hline & $\begin{array}{l}\text { Postural } \\
\text { Control and } \\
\text { Balance }\end{array}$ & Improved reactive strategies and BBS score reached 56 (maximum score) \\
\hline & $\begin{array}{l}\text { Aerobic } \\
\text { Capacity }\end{array}$ & Increased cycling and treadmill time and other intensity related parameters \\
\hline \multirow{2}{*}{ Activity Level } & $\begin{array}{l}\text { Functional } \\
\text { Abilities }\end{array}$ & $\begin{array}{c}\text { Ease, quick, less physical assistance, better confidence to move and without provoking pain or } \\
\text { fatigue after the activity }\end{array}$ \\
\hline & $\begin{array}{l}\text { Functional } \\
\text { Mobility }\end{array}$ & TUGT score $60 \%$ improvement \\
\hline \multirow{2}{*}{$\begin{array}{l}\text { Participation } \\
\text { Level }\end{array}$} & $\begin{array}{c}\text { Social } \\
\text { Participation }\end{array}$ & $\begin{array}{l}\text { started going out of the home to visit community places (10 times in } 12 \text { weeks as compared to } \\
7 \text { months of complete home-bound isolation), every time with less direct manual assistance, } \\
\text { could confidently face crowded places, and it did not provoke any of the disease symptoms. }\end{array}$ \\
\hline & & $\begin{array}{c}\text { For her ritual prayers she could not sit down in kneeling position. After intervention she could } \\
\text { sit more comfortably and ease of getting after wards made her concentrate more peacefully } \\
\text { during prayers. }\end{array}$ \\
\hline
\end{tabular}

Table 2: Patient showed improvement across all ICF domains.

\section{DISCUSSION}

Dermatomyositis is an inflammatory disabling neuromuscular disorder. The main goals when treating patients with DM are to improve function and prevent disability. This manuscript reviews the effectiveness and safety of an active rehabilitation program on all measures of disease activity, damage and patient-reported outcomes in a patient with adult dermatomyositis.
Use of core set measures has been documented in recent studies $[15,16]$. It needs close collaboration between the physician, Physical therapist and the patient in disease assessment. Patient was a responder to medical and Physical therapy intervention according to the International myositis assessment and clinical studies group definition of improvement. Patient met the IMACS improvement criteria for the entire core set measures and showed a major clinical response with 12 weeks of 
Physiotherapy intervention. Regarding effectiveness, muscle strength and MD Global activity are considered as top measures of improvement. It is important to mention that patient showed considerable improvement even in the more affected muscle groups. Resistive exercises given in this study were of moderate intensity and repetition for both supervised therapy program and HEP. Our findings on improvement in muscle function are in line with previous studies demonstrating effectiveness of resistive muscular training program in patients with chronic DM/PM [15-17].

\section{Potential Disease Modifying Effect}

At the beginning patient required high dose corticosteroid with other immunomodulatory therapy. It is important to reduce the dose to the smallest, most effective amount to prevent the adverse effects of chronic corticosteroid use. Progressively her physician administered a tapering dose of prescribed corticosteroids medication with no relapse of symptoms. Also, there was an improvement in physician and patient reported disease activity along with improvement in disease-specific biochemical markers (Table 1) indicating reduced inflammation. Alexanderson $\mathrm{H}$ and Lundberg IE (2012) [18] mentioned in a review that few studies have reported reduced clinical disease activity after resistance training in chronic phase of the disease. Some of these studies have combined clinical outcome measures with molecular studies on muscle and blood and provide new insights on effect of exercise on molecular pathogenesis. A detailed investigation of the effects of endurance exercise on molecular profile of skeletal muscles was done by Munters et al. [16]. This study showed that endurance exercise may activate an aerobic phenotype and promote muscle growth; genes related to capillary growth, mitochondrial biogene and muscle hypertrophy were up-regulated whereas simultaneously suppress the genes related to inflammation. Few studies showed down regulation of genes regulating systemic and local inflammation and fibrosis in muscle tissue after physical exercise and also inducing muscle growth and improving mitochondrial function. This encouraging data suggests that intensive exercise indeed could even be considered an anti-inflammatory treatment in adult patients with DM, but further studies are needed for deeper understanding of the molecular pathways which will confirm these findings and aid clinical research. Also, evidence-based data with long term follow-up may help us better treat the disease. Endurance exercise improves health and may reduce disease activity in patients with established PM/DM. This potentially could be mediated through improved aerobic fitness [16].

Previously it has been observed that although responding to treatment, a majority of patients with PM and DM develops sustained disability [19]. Up to one-third of patients will have mild to severe functional disability, and disability increases with disease duration. Alexanderson $\mathrm{H}$ in a review article [19] mentioned the need to further increase knowledge of mechanisms causing disability. Myositis leads to significant disease damage which refers to persistent changes in anatomy, physiology, pathology or function resulting from prior active disease, complications of cortico-steroid and immune-suppressive therapy, co-morbid conditions, etc. and often are irreversible and cumulative. It has been speculated that disability accumulates because of the inflammatory disease and complications of corticosteroid and immunosuppressive therapy. While inflammation causes muscle damage in the early phase, metabolic alterations such as impairment of oxidative metabolism seem to be responsible for the disability in the chronic phase of the disease. Dastmalchi M et al. [20] investigated muscle fibre type composition and muscle fiber area using biochemical and immunohistochemistry techniques in repeated muscle biopsy samples obtained from patients with chronic myositis before and after a 12 week moderate exercise program and in healthy controls. This study showed low muscle endurance in chronic PM or DM may be related to 
a low proportion of oxidative, slow-twitch type I fibres and supports the hypothesis of hypoxia in muscle tissue as a possible contributor to muscle weakness. An exerciseinduced increase in the mean fiber area and the percentage of type I oxygen dependent muscle fibres may contribute to improved muscle endurance and decreased muscle fatigue in patients with chronic PM or DM. Also, improved physical functioning on SF-36 was positively correlated with the proportion of type I fibers and type II fiber area. In a study by Alemo M et al. 2013 [16], exercise performance and aerobic capacity was found to be lower than healthy controls, and a 12 weeks of endurance training program increased cycling time, improved aerobic capacity and muscle mitochondrial enzyme activities. Authors emphasized the importance of endurance exercise in addition to immunosuppressive treatment. In a recent study by Bertolucci F et al. (2014) [21], a precocious fatigability and significantly higher values of hematic lactate were observed at rest and after the exercise (sub-maximal aerobic exercise on treadmill) suggesting an impaired muscle oxidative efficiency in PM/DM patients. In a study by Weisinger GF et al. [22], cardiorespiratory capacity expressed quantitatively as peak $\mathrm{VO}_{2}$ was diminished at $53 \%$ in patients with DM/PM as compared with age- and sex-matched controls. Author suggested that low $\mathrm{VO}_{2}$ at anaerobic threshold could contribute to limited functional capacity in daily activities. This data suggest that intensive aerobic exercise and resistance training could reduce disease activity and inflammation and improve muscle metabolism.

At the end of 4 weeks an improvement was observed in patient's cycling and tread mill time which further increased over a period of 12 weeks. Also, subjectively she reported improved tolerance to physical activities at home. This clinical improvement seems to correspond to improved muscle functions and increased aerobic capacity via above mentioned underlying mechanisms. These favorable results in endurance associated with improvement in perceived health and functional abilities may help to remediate the impairments underlying the disability in chronic phase of the disease but larger studies are needed to confirm. Also, physical exercise should be an integral part of therapy, particularly in view of the cardiopulmonary risk of these patients.

Specialists recommend physical therapy as an important part of treatment for muscle weakness associated with DM. Therapy should be started as soon as symptoms are under control. For her, medical treatment was started immediately (within 3 months) after acute flare up of symptoms and diagnosis whereas physiotherapy was started after 6 months. Delays in diagnosis often occur owing to a lack of recognition of disease presentation, or misdiagnosis, both of which can lead to muscle atrophy. Long standing symptoms before diagnosis and treatment have been reported in previous studies as poor predictors of outcome. In this case, diagnosis was delayed for several years resulting into significant muscle atrophy and disability by the time patient was referred to physical therapy. Initial part of physical therapy was focused to reverse the added effect of deconditioning apart from the effects of disease activity. In a study found that physical training begun 2 weeks - 3 weeks after an acute exacerbation of DM/PM seems to be safe and useful [23]. Muscle atrophy, due to lack of activity, may be partially prevented and level of disability can be decreased. Same author in other studies $[24,25]$ have reported that easy to moderate resistive exercise is well tolerated in people with active, recent-onset myositis and also safety of resistive home exercise program in recent onset active PM or DM. After 12 weeks of home exercise program along with the beneficial effects of significantly improved function and quality of life there were no signs of increased muscle inflammation. Marie et al. (2001) [26] in a longitudinal study found high mortality and morbidity related to $\mathrm{PM} / \mathrm{DM}$ emphasizing that management of DM/PM at an early stage is required. Physiotherapy if instituted during 
early recovery phase in this case could have yielded far better results in preventing muscle atrophy without increasing muscle inflammation.

Literature search conducted could identify very few studies which evaluated the long term influence of physical exercise in DM. A study [25] evaluated outcome of resistive home exercise in patients with recent-onset active PM or DM. This study involving a control group did not reveal any between-group differences in exercise effects on health, disability, and disease activity at the end of 24 weeks. However, in an open 2 year follow-up, exercise group maintained significant improvement in muscle performance and aerobic capacity compared to baseline, suggesting that sustained exercise may be of some benefit. Whereas only one study [17] by Weisinger (1998) documented continued benefits with along-term physical training program for 6 months and showed beneficial effects in muscle strength, patient's ADLs as well as cardiorespiratory fitness as compared to an untrained group. In a multicenter RCT [27] with 12 weeks of supervised endurance program, improvement in muscle strength was sustained up to 1 year whereas ADL score and SF-36 returned to baseline emphasizing the need for a continued exercise program. Therefore, it is essential to understand the largely unknown long term effects with future longitudinal studies.

\section{Health Enhancing Effect}

A number of studies have reported that patients with myositis have poor health-related QOL as measured using SF-36 and other generic measures [28-30]. In a study by Alexanderson H (2018) [27], SF-36 physical function correlated moderately with MMT-8 in patients with PM/DM. In addition to physical disabilities, these disorders may give rise to psychological consequences that negatively affect quality of life [28]. HAQ is a comprehensive patient-oriented outcome measure and assesses self-report functional status (disability). At the beginning patient had poor perceived health on SF-36 and considerable difficulty in functional abilities as assessed on HAQ (The Disability Index). It is observed that patient showed marked improvement across all components of SF-36 and the percentage change in HAQ was $23.33 \%$ indicating clinically significant health-enhancing effect after physical exercise intervention. Few studies reported improvement after physical exercise intervention. In this context, physical exercise is recommended to improve general health.

\section{Myalgia}

In a retrospective study using histopathological examinations, fasciitis (found in $75 \%$ patients with DM), rather than myositis, was associated with myalgia [31]. With this understanding, specific stretching exercises for thoracolumbar fascia incorporating breathing exercises were employed for her and pain in thoraco-lumbar and pelvic region subsided completely.

With regard to extra-skeletal muscle disease activity, patient had shortness of breath and frequent dry cough which significantly limited performance of her physical activities. These symptoms may indicate underlying interstitial lung disorder for which she was monitored with periodic clinical examination by the physician. With breathing exercises there was significant reduction in shortness of breath (On VAS reduction from 8.7 at baseline to 1.9 after 12 weeks) and subjectively patient reported feeling of better ability to control her symptoms. Relaxation/respiratory training help the patients to use diaphragmatic breathing in various positions to help strengthen this muscle and to learn to use movement more efficiently during exercise; transfers from bed to chair, chair to toilet, and activities of daily living.

\section{Task Specific Training}

It is important to mention that patient responded very well to task-specific approach for walking and getting up from floor activity. Subjectively she reported ease of movement and objectively there was an improvement in time required 
(initially 2.44 seconds for 20 repetitions with either leg with a pause in between due to cramps in legs - improved to 1.2 seconds with no cramps and better alignment and coordination of the body). However she continued to use grab bars in the toilet for getting up. Patient could go down on the staircase without manual assistance and became more confident. These improvements in functional activities can be explained in the context of strengthfunction relationship as the patient showed significant strength gain in axial and proximal muscles of lowerextremity.

The results involving resistive and aerobic exercise are encouraging for improvement in people with DM. A study [32] reported significant improvement regarding muscle function in the upper and lower limbs, walking distance and general health status in patients with stable, inactive PM and DM with HEP 12 weeks. There was improvement in strength, balance, performance in activities of daily living, and walking speed. In addition, improvement was seen in function, ability to perform activities of daily living, and health - related quality of life in adult DM with differing, individualized exercise programs. Most of these studies have used home exercise program.

The WHO has published the ICF as a unified nomenclature and framework to describe health and health-related conditions as well as the impact of a healthrelated condition on the affected individual. Relatively few authors have described interventional outcomes in myopathies based on the ICF terminology. In a study by Alexanderson H et al. (2007) [15] patients with chronic, stable PM and DM performed intensive resistive exercise program with beneficial effects on impairment and activity limitation. However, participation level (patient's disease impact on well-being) remained unchanged even after 7 weeks of exercise program. The reason for this is not clear. No other study could be identified in the literature which has evaluated participation level of patients with DM/PM. In the current study, patient's social participation markedly improved. This distinctive and meaningful outcome achieved could be attributed to a more holistic approach of intervention.

\section{Safety}

Previous studies have documented safety of physical exercises in PM/DM using various parameters viz. with muscle enzyme levels [23-25,33] muscle biopsy [15,25] and MRI of the muscles [25]. In a study on the benefits of intensive aerobic training [27], blood lactate levels were normal before and after treatment; moderate to intensive resistance and aerobic exercise is well tolerated by patients with chronic, stable low-activity myositis (DM and PM) without increased muscle inflammation. These studies reported no signs of increased disease activity indicating that supervised and/or HEP can be safely employed in patients with stable, inactive PM and DM. Patient presented with established, low disease activity. Clinically and from the investigation done periodically, it is observed that she showed better control of disease activity - viz. positive response to therapy with no adverse reaction and improved tolerance to exercise. According to the criteria used by Huber et al. [34], disease course is designated as monocyclic, in remission or chronic continuous as determined by clinical or biochemical parameters. It is difficult to designate this patient's disease course with accuracy as it was around 10 month's time from the diagnosis and that patient was on immunosuppressive treatment. However, from the progressively tapering dose of the medicines and stable disease activity maintained so far indicates a favorable disease course towards either 'monocyclic' or 'in remission' nature.

The importance of a closely monitored and adapted individualized program to ensure safety cannot be overemphasized. The clinical findings and also changes in disease-specific inflammatory biomarkers and responses to immunomodulatory therapy were considered on continual basis throughout the study period. 
Fatigue, pain and cognitive impact are other important disease consequences that have not been much addressed in the literature.

There has been concern that emotions and mental health can be very important in inflammatory skin diseases like DM. In previous studies, DM was found to have significantly worse impact on the emotional realm of QOL. As mentioned in the previous section of this article, many psychological problems were identified in this patient. Throughout the study period, this psychiatric comorbidity was taken into consideration. Therapist tried to deal with these problems by patient education to increase understanding of myositis and psychological counseling emphasizing on assurance, enhancing coping strategies, reducing concerns with treatment dependency (on medication), to empower patient to control disease activity by 'listening to your body', relaxation through meditation, breathing exercises, etc. Her overall understanding of disease condition, improved self-confidence to handle her bodily symptoms improved and reduced isolation-started going out outdoor to social places. After a continuous 1 month program she was assured of stable response of her body to exercises and thus was increasingly/more motivated. This can be documented in terms of ICF framework as improvement in personal factors. Living with a genetic or rare disease can impact the daily lives of patients and families. Patient advocacy organizations, social support groups and resources can help families navigate various aspects of living with a rare disease.

Emphasizing the necessity of patient involvement we report the qualitative inputs from the patient. Patient reported outcomes, described in her own words especially to mention are the comments that "I feel now I am living in my body".

From the summary of available research and the results of this case, it can be reported that exercise has emerged as an important part of the treatment for patients with
IIMs.During the last 15 years, intensive research activity has resulted in the accumulation of evidence to support the safety and efficacy of exercise in patients with both established and newly diagnosed inflammatory active disease [35-37]. However, considering the limitations of the available studies, there is a need for larger studies.

It is important to mention that majority of the studies cited here have clinical findings supported with biochemical and immune mediated parameters. We acknowledge that the primary purpose of this intervention was patient treatment and patient was not subjected to additional laboratory investigations periodically from research point of view.

\section{Significance and Innovation}

This manuscript illustrates the effectiveness and safety of an active rehabilitation program on all measures of disease activity, damage and patient-reported outcomes in a patient with adult dermatomyositis.

The effects have been assessed with validated objective and patient-reported outcome measures. IMACS recommended and Myositis-specific outcome measures were used to monitor the response to physical therapy and exercise.

Improvement has been seen with differing, individualized exercise programs in PM/DM. However, no recommended guidelines for physical therapy of DM are available. In a review de Sailles Parinelli (2009) [38], mentioned that the optimal exercise modality training remain to be determined in future studies. Previous studies have emphasized on the role of in resistive and endurance training. Apart from these exercises, this patient was given movement coordination, balance training, task-oriented functional training, etc. Thus, in the present study, a more comprehensive and optimal regimen was employed. These intervention strategies will provide clinicians with guidelines for developing protocols for DM. Also, it is 
important to mention that therapist should adopt a different approach should be sensitive to the psychological needs of these patients. To our knowledge this is the first study which reports effectiveness of a long term and combined supervised and home based exercise program. Being a single case study, results should be interpreted in the context of differences in the clinical presentations, natural history and responses to therapy. However, these results may be important to guide clinical decision making regarding physical therapy intervention for dermatomyositis and for further studies.

Results of this study should enhance our understanding to aid in clinical-decision making and developing guidelines for the development of therapies in the future; and couldimprove treatment and prognosis in patients with dermatomyositis.

\section{CONCLUSION}

Based on the previous research and from the results of this case presented here, it can be concluded that physical exercise is safe and effective in adult dermatomyositis. We suggest that physical exercise, adapted to disease activity and disability should be included in the rehabilitation of in all stages of dermatomyositis.

\section{CONSENT}

The patient providedinformed writtenconsent to participate in the study and for publication of this case report in any journal. IRB approval was not required for the described case.

\section{CONFLICT OF INTERESTS}

The author declares that there are no competing interests.

\section{FUNDING}

This study is self-funded and has not received any funding from external source.

\section{AUTHOR'S CONTRIBUTION}

The author is solely responsible for analysis and interpretation of the patient data; writing the manuscript; approving the final manuscript.

\section{ACKNOWLEDGEMENT}

The author would like to acknowledge the valuable contribution of the patient and her primary physician.

\section{REFERENCES}

1. Love L, Leff R, Fraser D, et al. (1991) A new approach to the classification of idiopathic inflammatory myopathy: Myositis-specific autoantibodies define useful homogeneous patient groups. Medicine 70(6): 360-374.

2. Dalakas MC (2015) Inflammatory muscle diseases. The New England Journal of Medicine 372(18): 1734-1747.

3. Meyer A, Meyer N, Gottenberg JE (2015) Incidence and prevalence of inflammatory myopathies: A systematic review. Rheumatology (Oxford) 54(1): 50-63.

4. Lundberg IE (2017) European league against rheumatism/American college of rheumatology classification criteria for adult and juvenile idiopathic inflammatory myopathies and their major subgroups. Arthritis \& Rheumatology 69(12): 2271-2282.

5. https://www.orpha.net/consor/cgi-bin/OC_Exp.php?

6. Meyer A et al. (2019) Review idiopathic inflammatory myopathies: State of the art on clinical practice guidelines. RMD Open 4(Suppl 1): e000784.

7. Regardt M, Henriksson EW, Alexanderson H, et al. (2011) Patients with polymyositis or dermatomyositis have reduced grip force and health-related quality of life in comparison with reference values: An observational study. Rheumatology (Oxford) 50(3): 578-585. 
http://www.tridhascholars.org | June-2021

8. Poulsen KB, Alexanderson H, Dalgård C, et al. (2017) Quality of life correlates with muscle strength in patients with dermato or polymyositis. Clinical Rheumatology 36(10): 2289-2295.

9. Alexanderson H (2016) Physical exercise as a treatment for adult and juvenile myositis. Journal of Internal Medicine 280(1): 75-96.

10. Rider LG, Giannini EH, Brunner HI, et al. (2004) International myositis assessment and clinical studies group. International consensus on preliminary definitions of improvement in adult and juvenile myositis. Arthritis \& Rheumatology 50(7): 2281-2290.

11. Miller FW, Rider LG, Chung YL, et al. (2001) International myositis outcome assessment collaborative study group. Proposed preliminary core set measures for disease outcome assessment in adult and juvenile idiopathic inflammatory myopathies. Rheumatology (Oxford) 40(11): 1262-1273.

12. Bonita R, Beaglehole R (1998) Modification of rankin scale: Recovery of motor function after stroke. Stroke 19(12): 14971500 .

13. Ware JE, Snow KK, Kosinski M, et al. (1989) SF-36 health survey: Manual and interpretation guide. Boston, MA: The Health Institute, New England Medical Center.

14. Aggarwal R, Rider LG, Ruperto N, et al. (2017) 2016 American college of rheumatology/European league against rheumatism criteria for minimal, moderate, and major clinical response in adult dermatomyositis and polymyositis: An International myositis assessment and clinical studies group/Paediatric rheumatology International trials organisation collaborative initiative. Annals of the Rheumatic Diseases 76(5): 792-801.

15. Alexanderson H, Dastmalchi M, Esbjornsson LM, et al. (2007) Benefits of intensive resistance training in patients with chronic polymyositis or dermatomyositis. Arthritis \& Rheumatology 57(5): 768-777.

16. Alemo M, Dastmalchi M, Katz A, et al. (2013) Improved exercise performance and increased aerobic capacity after endurance training of patients with stable polymyositis and dermatomyositis. Arthritis Research and Therapy 15(4): R83.

17. Wiesinger GF, Quittan M, Graninger M, et al. (1998) Benefit of 6 months long-term physical training in polymyositis/dermatomyositis patients. British journal of Rheumatology 37(12): 1338-1342.

18. Alexanderson H, Lundberg IE (2012) Exercise as a therapeutic modality in patients with idiopathic inflammatory myopathies. Current Opinions in Rheumatology 24(2): 201-207.

19. Alexanderson H (2009) Exercise effects in patients with adult idiopathic inflammatory myopathies. Current Opinions in Rheuamtology 21(2):158-163.

20. Dastmalchi M, Alexanderson H, Loell I, et al. (2007) Effect of physical training on the proportion of slow-twitch type I muscle fibers, a novel non-immune mediated mechanism for muscle impairment in polymyositis or dermatomyositis. Arthritis \& Rheumatology 57(7): 1303-1310.

21. Bertolucci F, Dalise S (2013) Abnormal lactate levels in patients with polymyositis and dermatomyositis: The benefits of a specific rehabilitative program. European Journal of Physical and Rehabilitation Medicine 50(2): 161-169.

22. Wiesinger GF, Quittan M, Nuhr M, et al. (2000) Aerobic capacity in adult dermatomyositis/polymyositis patients and healthy controls. Archives of Physical Medicine and Rehabilitation 81(1): 1-5.

23. Alexanderson H, Stenstrom CH, Lundberg IE (1999) Safety of a home exercise programme in patients with polymyositis and dermatomyositis: A pilot study. Rheumatology (Oxford England) 38(7): 608-611.

24. Alexanderson H, Stenstrom CH, Jenner G, et al. (2000) The safety of a resistive home exercise program in patients with recent onset active polymyositis or dermatomyositis. Scandinavian Journal of Rheumatology 29(5): 295-301. 
http://www.tridhascholars.org | June-2021

25. Alexanderson H, Munters A, Dastmalchi M, et al. (2014) Resistive home exercise in patients with recent-onset polymyositis and dermatomyositis - a randomized controlled single-blinded study with 2 year follow up. The Journal of Rheumatology 41(6): 1124-1132.

26. Marie I et al. (2001) Polymyositis and dermatomyositis: Short term and long term outcome and predictive factors of prognosis. Journal of Rheumatology 28(10): 2230-2237.

27. AlemoMunters L, Dastmalchi M, Andgren V, et al. (2013) Improvement in health and possible reduction in disease activity using endurance exercise in patients with established polymyositis and dermatomyositis: A multicenter randomized controlled trial with a 1-year open extension follow up. Arthritis Care \& Research (Hoboken) 65(12): 1959-1968.

28. Bronner IM, van der Meulen MF, de Visser M, et al. (2006) Long term outcome in polymyositis and dermatomyositis. Annals of the Rheumatic Diseases 65: 1456-1461.

29. Ponyi A, Borgulya G, Constantin T, et al. (2005) Functional outcome and quality of life in adult patients with idiopathic inflammatory myositis. Rheumatology 44(1): 83-88.

30. Sultan SM, Ioannou Y, Moss K, et al. (2002) Outcome in patients with idiopathic inflammatory myositis: Morbidity and mortality. Rheumatology 41(1): 22-26.

31. Kentaro Noda, Ken Yoshida, Taro Ukichi, et al. (2017) Myalgia in Patients with dermatomyositis and polymyositis is attributable to fasciitis rather than myositis: A retrospective study of 32 patients who underwent histopathological examinations. The Journal of Rheumatology 44(4): 482-487.

32. Alexanderson H, Regardt M, Ottoson C, et al. (2018) Muscle strengthened muscle endurance during the first year of treatment of polymyositis and dermatomyositis: A prospective study. The Journal of Rheumatology 45(4): 538-546.

33. Mattar MA, Gualano B, Perandini LA, et al. (2014) Safety and possible effects of low-intensity resistance training associated with partial blood flow restriction in polymyositis and dermatomyositis. Arthritis Research \& Therapy 16(5): 473.

34. Huber AM, Lang B, LeBlanc CM, et al. (2000) Medium and long term functional outcomes in a multicenter cohort of children with juvenile dermatomyositis. Arthritis \& Rheumatology 43(3): 541-549.

35. Alexanderson H, Lundberg IE (2005) The role of exercise in the rehabilitation of idiopathic inflammatory myopathies. Current Opinion in Rheumatology 17(2): 164-171.

36. Alexanderson H (2012) "Exercise in inflammatory myopathies, including inclusion body myositis". Current Rheumatology Reports 14: 244-251.

37. Lundberg I, Venecovsky J, Alexanderson H (2014) Therapy of myositis: Biological and physical. Current Opininon in Rheumatology 26(6): 704-711.

38. de Salles Painelli V, Gualano B, Giannini Artioli G, et al. (2009) The possible role of physical exercise on the treatment of idiopathic inflammatory myopathies. Autoimmunity Reviews 8(5): 355-359. 